\title{
Prueba de Autoestima para Adolescentes ${ }^{*}$
}

The self-esteem test for adolescents

Recibido: noviembre 12 de 2009 ～Revisado: abril 21 de 2010 ～Aceptado: mayo 18 de 2010

\author{
JOAQUÍN CASO NIEBLA ** \\ Universidad Autónoma de Baja California, México \\ LAURA HERNÁNDEZ-GUZMÁN **** \\ Universidad Nacional Autónoma de México, México \\ MANUEL GONZÁLEZ-MONTESINOS ${ }^{* * * * *}$ \\ Universidad de Sonora, México
}

\section{RESUMEN}

El presente estudio se propuso indagar sobre la validez de constructo de la adaptación a la Prueba de Autoestima para Adolescentes (PAA) y actualizar los parámetros psicométricos resultantes de estudios anteriores. Respondieron el instrumento 1581 estudiantes de ambos sexos (850 mujeres y 731 hombres) de una institución pública de educación media superior de la Ciudad de México. Mediante el empleo de la metodología de validez cruzada se determinó que el modelo de medida que subyace a la PAA ajustó satisfactoriamente, lo que confirma una estructura de cuatro factores: Cogniciones sobre sí mismo, Cogniciones de competencia, Relación familiar y Enojo. Los hallazgos del presente estudio se suman a los datos generados en torno a la validez de contenido, de criterio y de constructo de la PAA. Palabras clave autores

Autoestima, adolescentes, modelamiento estructural, validez, estudio instrumental. Palabras clave descriptores

Adolescent psychology, psicometría, test psicológico, diferencia individual, comportamiento social, relaciones interpersonales, afectividad.

Para citar este artículo. Caso, N. J., HernándezGuzmán, L. \& González-Montesinos, M. (2011). Prueba de Autoestima para Adolescentes. Universitas Psychologica, 10 (2), 535-543.

* Artículo de investigación.

*** Carretera Tijuana-Ensenada km. 103, C.P 22830 Col. Playitas, Ensenada, B.C., México. E-mail: jcaso@uabc.mx

**** Av. Universidad 3004, Col. Copilco Universidad, Delegación Coyoacán, C.P. 04510, México, Distrito Federal, México.E-mail: lher@servidor.unam.mx. Website: http://aurahernandezguzman.com/

***** Blvd. Luis Encinas y Rosales S/N, Col. Centro, Hermosillo, Sonora, México.E-mail: manuelgm4@ gmail.com

\section{A B S T R A C T}

The present study intended to explore construct validity of the Self-esteem Test for Adolescents and update psychometric properties found in previous studies. 1581 Mexican students (850 women and 731 men) of a public high school in Mexico City responded to the scale. The sample was split randomly in half. EFA was applied using one sample's data, and CFA to the other sample's data. The model, assumed to underlie responses to the Self-esteem Test for Adolescents, satisfactorily fit the data, confirming a structure of 4 factors: self-cognitions, competence cognitions, family relations and rage. Results of the present study corroborate previous data concerning content, criterion-related and construct validity of the Selfesteem Test for Adolescents.

Key words authors

Self-esteem, Adolescents, SEM, Validity, Instrumental Study.

Key words plus

Adolescent Psychology, Psychometrics, Psychological Tests, Individual Differences, Social Behaviour, Interpersonal Relations, Emotions. 
La autoestima forma parte del proceso de desarrollo del individuo, por lo que su conceptuación ha dependido de los contextos con los que ha interactuado la persona a lo largo de su vida, y en el momento del ciclo vital en el que se encuentre. En la adolescencia, por ejemplo, predominan los contextos familiar, escolar y de los compañeros. No sorprende, entonces, que una buena parte de la investigación sobre autoestima adolescente encuentre reiteradamente que se asocia en forma positiva con el rendimiento exitoso en el contexto escolar (DuBois, Bull, Sherman \& Roberts, 1998; Hoge, Smit \& Crist, 1995) y familiar (Franklin \& Streeter, 1995), y negativamente con la disfunción personal, como la depresión (Litten, 1999) y el consumo de sustancias (Dishion, Kavanagh, Schneiger, Neilson \& Kaufman, 2002), entre otras.

A pesar de tratarse de un constructo de amplia utilización y del dominio del vulgo, ha sido limitado el número de modelos que reconozcan su implicación en los planos de funcionamiento cognitivo, emocional y conductual de cada persona, a la vez que permitan construir un puente de investigación que arroje alguna luz sobre su carácter evolutivo, multidimensional, e interactivo. Los modelos tradicionalmente propuestos han coincidido en explicarla en términos de una autoestima global adolescente. Autoestima global que se integra, según Harter (1983), a partir de dos dimensiones básicas: la apariencia física y la competencia escolar. De acuerdo con otras posturas, como la de Shavelson, Hubner y Stanton (1976), se integraría también en dos dimensiones, académica y una no académica, pero reconocen la existencia de componentes emocionales, sociales y físicos (personales) de la autoestima no académica.

Es relativamente reciente la asociación entre autoestima y afrontamiento, aunque no es nueva la propuesta de atribuir el desarrollo de la autoestima a la influencia del ambiente, a la historia de éxito del niño, y a la forma en que interpreta los éxitos y los fracasos (Coopersmith, 1967). De acuerdo con Frydenberg (1997), al momento de afrontar una situación determinada, el adolescente interpreta y evalúa las características del ambiente circundante, ya sea como una amenaza, un conflicto, un peligro o un desafío, o bien como inocuas o inofensivas. La autoestima desempeña así un papel importante en la valoración inicial que hace el adolescente, en forma de cogniciones, de sí mismo ante las demandas situacionales (Bednar, 1991). A esas cogniciones las acompañan emociones concomitantes que definen su desempeño. Como constructo que explica el funcionamiento adaptativo, la autoestima ha merecido atención por parte de teóricos e investigadores. El análisis de su abordaje teórico (Fajardo-Vargas, Hernández-Guzmán \& Caso-Niebla, 2001) destaca, precisamente, la evaluación que realiza la persona de sí misma y de su desempeño personal al interpretar las características psicológicas de las situaciones que afronta cotidianamente. Dependiendo de su respuesta, ya sea ésta de evitación o activa orientada a la solución de problemas, el adolescente evaluará su propia actuación. La retroalimentación, ya sea interna o externa, proporcionada por sus experiencias, pasará a formar parte de su historia personal de éxitos y fracasos, e irá moldeando su autoestima. Tomando en cuenta la importancia del contexto, de las cogniciones asociadas a esa valoración y de las emociones que matizan a la autoestima como el componente valorativo/emocional del afrontamiento, el presente trabajo propone cuatro dimensiones principales de la autoestima adolescente: la académica, la personal, la familiar y la emocional.

El modelo propuesto reconoce el componente valorativo de las manifestaciones conductuales, cognitivas y emocionales de la autoestima, a la luz de los contextos vitales en los que se desempeña típicamente el adolescente. Por otra parte, la escasez de instrumentos en español, para medir la autoestima adolescente, cede relevancia al instrumento derivado de este modelo, la Prueba de Autoestima para Adolescentes (Caso \& Hernández-Guzmán, 2001) que cuenta ya con datos para población mexicana y, como resultado de diversos estudios, representa una alternativa válida y confiable. Ha alcanzado porcentajes de varianza explicada superior a $54 \%$ y su estructura factorial coincide de un estudio a otro (Caso \& Hernández-Guzmán, 2001; Celis, Medina-Mora, Caso, Osorno \& Stack, 2005; Jiménez \&Yáñez, 2006). 
Es, sin embargo, todavía una asignatura pendiente indagar sobre su validez de constructo y actualizar los parámetros psicométricos arrojados por estudios anteriores. Reconociendo los alcances del análisis factorial confirmatorio en la generación de datos en materia de validez de constructo, el presente estudio se ha propuesto corroborar si los ítems que componen la PAA miden el mismo rasgo latente, autoestima. También se propuso someter a prueba el modelo de cuatro dimensiones que intenta explicar la autoestima, y, por último, corroborar su consistencia interna, establecida mediante alfa de Cronbach, que describe el cálculo de la confiabilidad basado en la correlación promedio entre los ítems dentro del instrumento (Nunnally $\&$ Bernstein, 1994).

\section{Método}

\section{Participantes}

Participaron 1581 estudiantes de una institución de educación media superior pública de la Ciudad de México, 850 mujeres (53\%) y 731 hombres (47 $\%)$, con una edad promedio de 17.4 años $( \pm 1.3)$. Sus edades fluctuaban entre los 15 y los 23 años. De éstos, 634 (40.1\%) estudiaban el primer año escolar, 334 (21.1\%) el segundo y 613 (38.8\%) el tercero, 1018 en el turno matutino $(64.4 \%)$ y 563 (35.6\%) en el turno vespertino.

Se seleccionó a los participantes mediante el método de muestreo aleatorio simple y se consideró como unidad de muestreo al grupo escolar de adscripción, lo que entrañó la participación de estudiantes pertenecientes a 51 grupos escolares. Los estudiantes fueron seleccionados mediante el método de muestreo aleatorio sistemático, considerando como unidad de muestreo al grupo escolar de adscripción.

\section{Instrumentos}

Se aplicó la Prueba de Autoestima para Adolescentes (Caso \& Hernández-Guzmán, 2001), escala conformada por 21 reactivos con cinco opciones de respuesta tipo Likert, con un índice de confia- bilidad alfa $=0.88$, y agrupados en cuatro factores: Cogniciones sobre sí mismo, Cogniciones de competencia, Relación familiar y Enojo. Los reactivos cuentan con cinco opciones de respuesta (siempre, usualmente, algunas veces, rara vez, nunca) y se califican con 5 las respuestas que impliquen expresiones relacionadas con una alta autoestima y con 1 las que impliquen baja autoestima. Una calificación alta en la escala, representa una mayor autoestima.

\section{Procedimiento}

Una vez depurada la base de datos, se procedió a realizar los siguientes tipos de análisis: a) análisis de reactivos utilizando la metodología de teoría de respuesta al ítem de Rasch-Masters, b) análisis factorial exploratorio, c) análisis factorial confirmatorio y d) obtención de índices de consistencia interna. Como primer paso para la aplicación del análisis factorial, se procedió a dividir la base de datos en dos submuestras determinadas en forma aleatoria. Una, representada por $n=744$ casos, se analizó mediante el análisis factorial exploratorio y otra, representada por $n=804$ casos, a la que se aplicó el análisis factorial confirmatorio. Para ello, se requirió del apoyo del módulo que, para este propósito, cuenta el SPSS. La aleatorización produce una situación en la que ambas submuestras son equivalentes en todos los aspectos relevantes, excepto en el procedimiento que se emplea para analizarlas. El empleo de esta técnica se denomina validación cruzada, tiene la ventaja de que se obtiene una validación concurrente desde dos metodologías distintas, pero complementarias que establece - con sustento matemático y empírico- la estructura dimensional del instrumento bajo análisis.

\section{Resultados}

\section{Análisis de reactivos}

Como procedimiento de rutina previo al análisis de dimensionalidad, se obtuvieron los estadísticos básicos asociados al conjunto de reactivos que conforman la escala (Tabla 1). El resultado de este aná- 
lisis permitió observar que los veintiún reactivos de la escala, presentan coeficientes de correlación punto biserial superiores a 0.20 y una correlación promedio de 0.53 , valores aceptables de acuerdo con diversos sistemas de referencia. El coeficiente punto biserial proporciona el grado de asociación entre el ítem y el puntaje total de una prueba (Tristán \& Vidal, 2006), donde una correlación positiva indica que el grupo con mayor puntaje en la variable o atributo evaluado, representado por el
$50 \%$ de los sujetos, presenta una mayor media en las puntuaciones totales que el grupo bajo.

Posteriormente, se obtuvieron los valores de los estadígrafos de ajuste para los reactivos que conforman la escala, observándose que la totalidad de los reactivos satisfacen los criterios de bondad de ajuste interno y externo de entre 0.50 y 1.50 (Linacre, 2007), por lo que se concluye que todos los ítems se comportaron en el análisis, según las expectativas del modelo Rasch Masters. Sólo los

TABLA 1

Medias, desviaciones estándar y correlaciones punto biseriales de los reactivos de la Prueba de Autoestima para Adolescentes

\begin{tabular}{lcccc}
\hline \multicolumn{1}{c}{ Reactivo } & N & Media & Desv. estándar & Correlación punto biserial \\
\hline 1.Me gusta como soy & 1581 & 4.26 & 0.65 & 0.59 \\
2.Hago enojar a mis padres & 1576 & 3.02 & 0.66 & 0.44 \\
3.Me siento fracasado & 1573 & 4.12 & 0.84 & 0.67 \\
4.Estoy feliz de ser como soy & 1573 & 4.36 & 0.76 & 0.65 \\
5.Si me enojo con un amigo (a) lo(a) insulto & 1577 & 3.93 & 1.13 & 0.26 \\
6.Me siento bien cuando estoy con mi familia & 1577 & 4.40 & 0.72 & 0.52 \\
7.Mi familia está decepcionada de mí & 1571 & 4.49 & 0.77 & 0.55 \\
8.Tengo una de las mejores familias de todo el mundo & 1573 & 4.18 & 1.07 & 0.51 \\
9.Soy muy lento para realizar mi trabajo escolar & 1577 & 3.47 & 1.01 & 0.44 \\
10.Soy tonto (a) para hacer los trabajos de la escuela & 1572 & 4.46 & 0.70 & 0.53 \\
11.Estoy orgulloso (a) del trabajo que hago en la escuela & 1573 & 3.80 & 0.84 & 0.58 \\
12.Soy malo para muchas cosas & 1570 & 3.85 & 0.75 & 0.59 \\
13.Me enojo cuando mis padres no me dejan hacer lo que & 1571 & 2.91 & 1.29 & 0.41 \\
yo quiero & & & & 0.73 \\
14.Estoy orgulloso (a) de mí & 1574 & 4.35 & 0.77 & 0.68 \\
15.Les echo la culpa a otros de cosas que yo hago mal & 1574 & 4.20 & 0.75 & 0.37 \\
16.Pienso que mis padres serían felices si yo fuera diferente & 1568 & 4.02 & 1.51 & 0.63 \\
17.Soy un(a) buen(a) amigo(a) & 1572 & 4.37 & 0.68 & 0.33 \\
18.Tengo una mala opinión de mí mismo & 1573 & 4.20 & 1.05 & 0.69 \\
19.Me gustaría ser otra persona & 1567 & 4.32 & 1.08 & 0.64 \\
20.Me gusta la forma como me veo & 1568 & 3.98 & 0.96 & 0.59 \\
21.Siento ganas de irme de mi casa & 1574 & 4.34 & 0.96 & \\
\hline
\end{tabular}

Fuente: elaboración propia 
reactivos 2 y 8 presentan valores fuera de los umbrales críticos (Tabla 2).

\section{Análisis factorial exploratorio}

Con la submuestra de la base original $(n=744)$, seleccionada aleatoriamente, se realizó el análisis factorial exploratorio mediante el método de ex- tracción de máxima verosimilitud. A este respecto, se registró un agrupamiento de los reactivos en cuatro factores que en conjunto explican el 55.5 $\%$ de la varianza: $32.6 \%$ por el factor Cogniciones sobre sí mismo (F1), $10.1 \%$ por el factor Cogniciones de competencia (F2), $6.9 \%$ por el factor Relación familiar (F3) y $5.9 \%$ por el factor Enojo (F4) (Tabla 3).

TABLA 2

Estadígrafos de ajuste del modelo Rasch para los reactivos de la Prueba de Autoestima para Adolescentes

\begin{tabular}{|c|c|c|c|c|c|c|c|}
\hline \multirow{2}{*}{ Reactivo } & \multirow{2}{*}{$\mathrm{N}$} & \multirow{2}{*}{ Medida } & \multirow{2}{*}{ Error } & \multicolumn{2}{|c|}{ INFIT } & \multicolumn{2}{|c|}{ OUTFIT } \\
\hline & & & & MNSQ & ZEMP & MNSQ & ZEMP \\
\hline 7 & 1571 & 1.45 & 0.04 & 1.29 & 0.6 & 1.19 & 0.4 \\
\hline 10 & 1572 & 1.39 & 0.03 & 1.14 & 0.3 & 1.09 & 0.2 \\
\hline 21 & 1574 & 1.19 & 0.03 & 1.29 & 0.7 & 1.23 & 0.5 \\
\hline 19 & 1567 & 1.17 & 0.03 & 1.44 & 1.0 & 1.40 & 0.9 \\
\hline 18 & 1573 & 1.00 & 0.03 & 1.23 & 0.6 & 1.24 & 0.6 \\
\hline 15 & 1574 & 1.00 & 0.03 & 0.81 & -0.6 & 0.78 & -0.6 \\
\hline 3 & 1573 & 0.90 & 0.03 & 0.85 & -0.5 & 0.86 & -0.4 \\
\hline 16 & 1568 & 0.79 & 0.03 & 1.35 & 1.0 & 1.28 & 0.8 \\
\hline 5 & 1577 & 0.69 & 0.03 & 1.00 & 0.0 & 0.98 & -0.1 \\
\hline 12 & 1570 & 0.61 & 0.02 & 0.61 & -1.5 & 0.62 & -1.4 \\
\hline 9 & 1577 & 0.27 & 0.02 & 0.69 & -1.3 & 0.69 & -1.3 \\
\hline 2 & 1576 & -0.09 & 0.02 & 0.40 & -3.1 & 0.40 & -3.0 \\
\hline 13 & 1571 & -0.17 & 0.02 & 0.79 & -0.9 & 0.80 & -0.8 \\
\hline 11 & 1573 & -0.79 & 0.02 & 0.95 & -0.2 & 1.06 & 0.2 \\
\hline 20 & 1568 & -0.97 & 0.03 & 1.09 & 0.3 & 1.18 & 0.5 \\
\hline 8 & 1573 & -1.20 & 0.03 & 1.41 & 1.1 & 1.53 & 1.3 \\
\hline 1 & 1581 & -1.31 & 0.03 & 0.94 & -0.2 & 1.04 & 0.1 \\
\hline 14 & 1574 & -1.44 & 0.03 & 1.26 & 0.6 & 1.43 & 0.9 \\
\hline 4 & 1573 & -1.47 & 0.03 & 1.27 & 0.7 & 1.44 & 1.0 \\
\hline 17 & 1572 & -1.47 & 0.03 & 1.02 & 0.1 & 1.10 & 0.3 \\
\hline 6 & 1577 & -1.53 & 0.03 & 1.30 & 0.7 & 1.50 & 1.0 \\
\hline Media & 1573 & 0.00 & 0.03 & 1.05 & 0.0 & 1.09 & 0.0 \\
\hline D.E & 3 & 1.08 & 0.00 & 0.28 & 1.0 & 0.30 & 1.0 \\
\hline
\end{tabular}

Fuente: elaboración propia 


\section{TABLA 3}

Estructura factorial de la Prueba de Autoestima para Adolescentes (Método de extracción: Máxima verosimilitud)

\begin{tabular}{|c|c|c|c|c|}
\hline Reactivo & $\mathrm{F} 1$ & $\mathrm{~F} 2$ & F3 & $\mathrm{F} 4$ \\
\hline 4.Estoy feliz de ser como soy & 0.826 & & & \\
\hline 1.Me gusta como soy & 0.824 & & & \\
\hline 14.Estoy orgulloso (a) de mí & 0.652 & & & \\
\hline $\begin{array}{l}\text { 20.Me gusta la forma como } \\
\text { me veo }\end{array}$ & 0.634 & & & \\
\hline $\begin{array}{l}\text { 18. Tengo una mala opinión } \\
\text { de mí mismo }\end{array}$ & 0.532 & & & \\
\hline $\begin{array}{l}\text { 19.Me gustaría ser otra per- } \\
\text { sona }\end{array}$ & 0.503 & & & \\
\hline $\begin{array}{l}\text { 10.Soy tonto (a) para hacer } \\
\text { los trabajos de la escuela }\end{array}$ & & 0.571 & & \\
\hline $\begin{array}{l}\text { 9.Soy muy lento para realizar } \\
\text { mi trabajo escolar }\end{array}$ & & 0.565 & & \\
\hline 3.Me siento fracasado & & 0.531 & & \\
\hline $\begin{array}{l}\text { 12.Soy malo para muchas } \\
\text { cosas }\end{array}$ & & 0.485 & & \\
\hline $\begin{array}{l}\text { 11.Estoy orgulloso (a) del tra- } \\
\text { bajo que hago en la escuela }\end{array}$ & & 0.422 & & \\
\hline $\begin{array}{l}\text { 6.Me siento bien cuando } \\
\text { estoy con mi familia }\end{array}$ & & & 0.746 & \\
\hline $\begin{array}{l}\text { 8. Tengo una de las mejores } \\
\text { familias de todo el mundo }\end{array}$ & & & 0.690 & \\
\hline $\begin{array}{l}\text { 7.Mi familia esta decepciona- } \\
\text { da de mí }\end{array}$ & & & 0.516 & \\
\hline $\begin{array}{l}\text { 21.Siento ganas de irme de } \\
\text { mi casa }\end{array}$ & & & 0.456 & \\
\hline $\begin{array}{l}\text { 16.Pienso que mis padres } \\
\text { serían felices si yo fuera di- } \\
\text { ferente }\end{array}$ & & & 0.381 & \\
\hline 2.Hago enojar a mis padres & & & & 0.519 \\
\hline $\begin{array}{l}\text { 13.Me enojo cuando mis } \\
\text { padres no me dejan hacer lo } \\
\text { que yo quiero }\end{array}$ & & & & 0.472 \\
\hline $\begin{array}{l}\text { 15.Les echo la culpa a otros } \\
\text { de cosas que yo hago mal }\end{array}$ & & & & 0.433 \\
\hline $\begin{array}{l}\text { 5.Si me enojo con un amigo } \\
\text { (a) lo(a) insulto }\end{array}$ & & & & 0.415 \\
\hline
\end{tabular}

Varianza explicada: Total $=55.5 \%$, Factor $1=32.6 \%$, Factor $2=10.1 \%$, Factor $3=6.9 \%$ y Factor $4=5.9 \%$.

Fuente: elaboración propia
El presente arreglo excluyó al reactivo diecisiete "soy un(a) buen(a) amigo(a)" que presentó una carga factorial inferior a 0.30. La estructura factorial observada convergió en 6 iteraciones mediante rotación Varimax.

\section{Análisis factorial confirmatorio}

Por otra parte, para el análisis factorial confirmatorio se utilizó la segunda submuestra $(n=804)$. El análisis siguió la secuencia de modelamiento estructural de LISREL 8.57 (Joreskog \& Sorbom, 2001), en la que, primeramente, se genera una matriz de correlaciones policóricas a la que, posteriormente, se implementa el análisis confirmatorio correspondiente. Este paso es indispensable ya que los valores observados en las variables son de tipo categórico-ordinal.

Los resultados del análisis confirmatorio para el modelo de medida, revelan, en general, índices de bondad de ajuste aceptables. Se confirmó el modelo de 20 ítems con cuatro factores correlacionados, presentando una Chi cuadrada $=(\mathrm{gl}$ $=164) 623.70$, con una probabilidad asociada de 0.000 , y una chi-cuadrada normada $=3.80$. A pesar de que no existen lineamientos claros acerca del valor aceptable de la chi-cuadrada normada, Bollen (1989) ha sugerido que un valor hasta 5.0 indica un ajuste razonable. El valor del índice CFI (índice de ajuste comparativo de Bentler) y del Índice de ajuste incrementado (IFI, por sus siglas en inglés), cuyos valores deben ubicarse por encima de 0.90, mostraron un buen ajuste, pues resultaron de 0.94. En tanto, el índice RMSEA (el error cuadrático medio de aproximación) resultó de 0.059 , mostrando un buen ajuste ya que los valores oscilan entre 0.05 y 0.08. (Hair, Anderson, Tathman \& Black, 1999). Con base en estos resultados, puede concluirse que se ha representado la estructura factorial del instrumento observada en el Análisis factorial Exploratorio practicado a la primer submuestra. El modelo resultante se presenta en la Figura 1.

La interpretación concatenada de los resultados de los análisis exploratorio y confirmatorio, permite establecer que la estructura dimensional 
del instrumento Prueba de Autoestima para Adolescentes corresponde a la estructura propuesta por el modelo teórico.

\section{Consistencia interna}

Finalmente, el análisis de confiabilidad de los 20 reactivos que conforman la prueba, arrojó un alfa de 0.88 , mientras que sus factores presentaron valores de $0.88,0.75,0.75$ y 0.58 (Tabla 4).
TABLA 4

Índices de consistencia interna de la Prueba de $\mathrm{Au}$ toestima para Adolescentes

\begin{tabular}{|c|c|c|}
\hline Factor & $\begin{array}{l}\text { No. de } \\
\text { reactivos }\end{array}$ & $\begin{array}{l}\text { Alpha de } \\
\text { Cronbach }\end{array}$ \\
\hline Factor 1: Percepción de sí mismo & 6 & 0.88 \\
\hline $\begin{array}{l}\text { Factor 2: Percepción de compe- } \\
\text { tencia }\end{array}$ & 5 & 0.75 \\
\hline Factor 3: Relación familiar & 5 & 0.75 \\
\hline Factor 4: Manejo de emociones & 4 & 0.58 \\
\hline Escala total & 20 & 0.88 \\
\hline
\end{tabular}

Fuente: elaboración propia

\section{Figura 1}

Modelo de medida de la Prueba de Autoestima para Adolescentes.

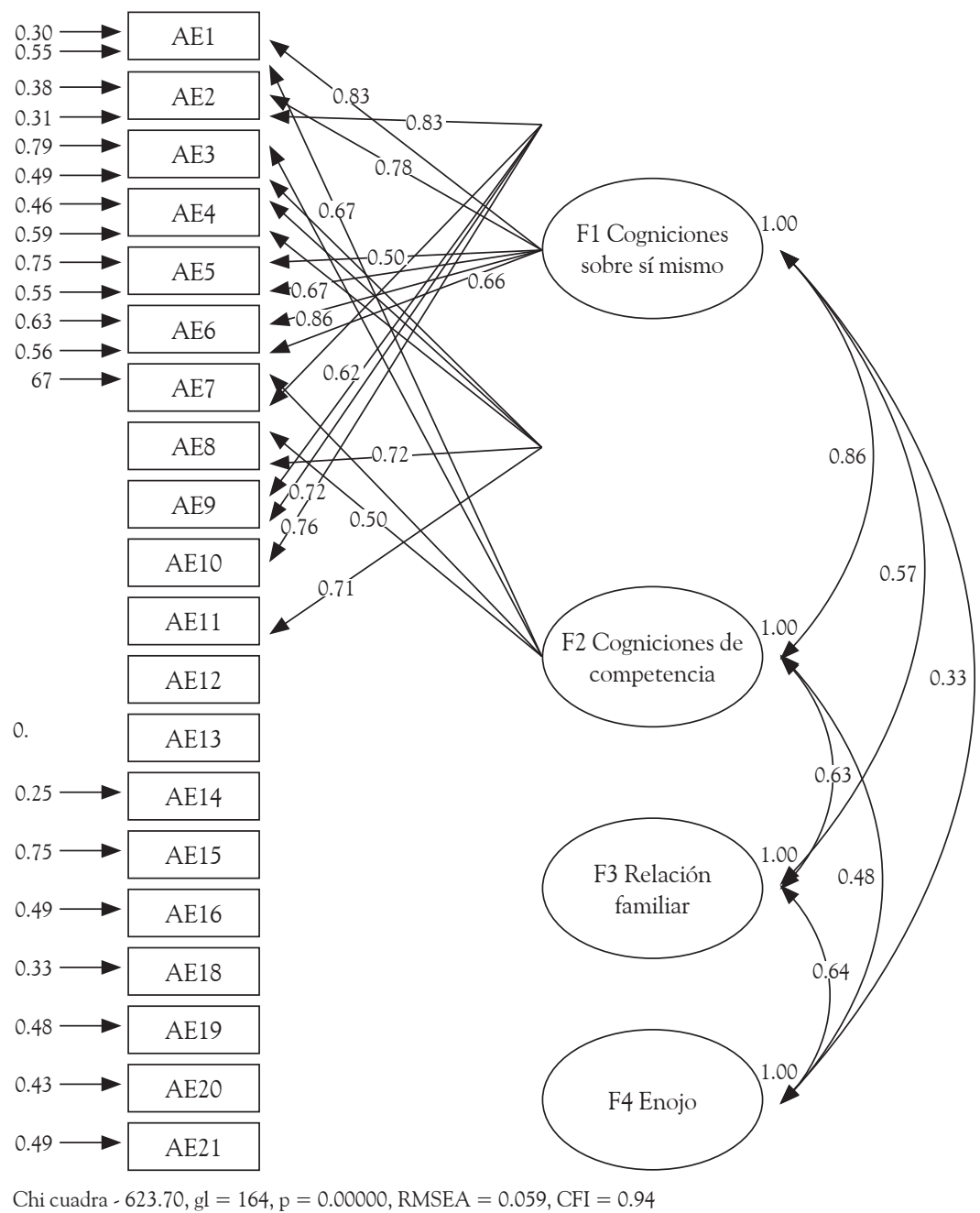

Chi cuadra $-623.70, \mathrm{gl}=164, \mathrm{p}=0.00000, \mathrm{RMSEA}=0.059, \mathrm{CFI}=0.94$

Fuente: elaboración propia 


\section{Discusión}

El presente estudio se propuso analizar los reactivos utilizando la metodología de Teoría de Respuesta al Item (TRI) de Rasch-Masters, para investigar si los reactivos que componen la Prueba de Autoestima para Adolescentes miden el mismo rasgo latente: autoestima. Por otra parte, buscaba someter a prueba la estructura factorial del modelo de medida encontrada por Caso y Hernández-Guzmán (2001) de 4 factores correlacionados, y explorar si confirma también la propuesta teórica. También, perseguía indagar su consistencia interna mediante alfa de Cronbach.

De acuerdo con la TRI, en la medida en la que la persona sea más afín al constructo autoestima, tendrá mayor probabilidad de responder aceptablemente a cualquier ítem de la prueba. Los resultados obtenidos del análisis de reactivos utilizando la metodología de teoría de respuesta al ítem de RaschMasters, sugieren que los ítems que componen la prueba miden el rasgo latente autoestima.

Respecto al segundo propósito, el análisis confirmatorio, para el modelo de medida de 20 ítems, resultado del análisis factorial exploratorio, revela en general buenos índices de bondad de ajuste. Los valores observados producto del análisis factorial confirmatorio en atención a lo propuesto por la metodología de validación cruzada, indican que el modelo propuesto ajusta a los datos de una forma razonable lo que implica que la estructura factorial del instrumento se ha representado de una forma técnicamente aceptable.

El RMSEA (el Error Cuadrático Medio de Aproximación) indicó un buen ajuste. Los valores de los parámetros estimados junto con los índices de bondad de ajuste ofrecen datos adicionales que apoyan la validez de constructo del instrumento respecto, no sólo a la estructura revelada por el análisis factorial exploratorio, sino con la estructura encontrada en los estudios previos (Caso \& Hernández-Guzmán, 2001; Celis et al., 2005; Jiménez \& Yáñez, 2006).

La solución factorial que especifica 4 factores correlacionados, refleja claramente 4 dimensiones que, de acuerdo con el modelo propuesto, son re- levantes durante la adolescencia: (1) la personal, representada por el factor Cogniciones sobre sí mismo, (2) la interacción con el contexto escolar, predominante durante esa etapa de la vida, representada por el factor Cogniciones de competencia, (3) la interacción con el contexto familiar, representada por el factor Relación familiar, y por último (4) la dimensión emocional, caracterizada en la adolescencia por el enojo, representada por el factor Enojo. Las dos primeras dimensiones corroboran las propuestas por Harter (1983), sin embargo, este modelo añade dos dimensiones: las relaciones que establece el adolescente con su contexto familiar y sus emociones. Este último factor, Enojo, presentó una confiabilidad baja. Sin embargo, se mantuvo, pues desde la perspectiva de la TRI, podría afirmarse que sus reactivos miden el constructo autoestima.

Los resultados del presente estudio con adolescentes, favorecen la estructura de 4 factores relacionados. La eliminación de un (1) ítem que no cumplió con las expectativas del modelo tiene sentido teórico, ya que los ítems relativos al contexto del grupo de iguales se distribuyeron en los diversos factores de la prueba. Dado que la interacción con el contexto de los pares o grupo de iguales reviste bastante importancia en la adolescencia, habrá que investigarlo en estudios futuros.

Los datos observados en torno a la aplicación de este instrumento a una muestra de estudiantes mexicanos, proporciona el soporte necesario para su utilización. La naturaleza de sus ítems, la estabilidad de su estructura factorial, el porcentaje de varianza explicada, su adecuación con los marcos conceptuales prevalecientes, los índices de confiabilidad y los datos de validez, así lo sugieren.

El índice de consistencia interna registrado por el instrumento y el porcentaje de varianza explicada en su versión para adolescentes, superan las observadas en aplicaciones previas (Caso \& Hernández-Guzmán, 2001).

En conclusión, los hallazgos del presente estudio se suman a los datos de investigación antecedente en torno a la validez de contenido, de criterio y de constructo de la Prueba de Autoestima para Adolescentes. Lo anterior constituye una 
aportación significativa a la medición de dominios afectivos, en adolescentes mexicanos, en contextos educativos.

\section{Referencias}

Bednar, R. L. (1991). Self-esteem: Paradoxes and innovation in clinical theory and practice. Washington DC: American Psychological Association.

Bollen, K. A. (1989). Structural equations with latent variables. New York: John Wiley \& Sons.

Caso, J. \& Hernández-Guzmán, L. (2001). iSon contextos medioambientales los que definen las dimensiones de la autoestima en niños y adolescentes? Revista Mexicana de Psicología, 18 (1), 229-237.

Celis, M., Medina-Mora, P., Caso-Niebla, J., Osorno, P. \& Martínez-Stack, J. (2005). Alumnos con rezago escolar en el bachillerato de la Universidad Nacional Autónoma de México. En Memorias del I Congreso Nacional de Orientación Educativa. UNAM: Dirección General de Orientación y Servicios Educativos.

Coopersmith, S. (1981). The antecedents of self-esteem. Palo Alto, CA: Consulting Psychologists Press.

Dishion, T. J., Kavanagh, K., Schneiger, A., Neilson, S. \& Kaufman, N. K. (2002). Preventing early adolescent substance use: A family-centered strategy for the public middle school. Prevention Science, 3 (3), 191-201.

DuBois, D. L., Bull, C. A., Sherman, M. D. \& Roberts, M. (1998). Self-esteem and adjustment in early adolescence: A social-contextual perspective. Journal of Youth and Adolescence, 27 (5), 557-583.

Fajardo-Vargas, V., Hernández-Guzmán, L. \& Caso-

Niebla, J. (2001). La autoestima y su relación con la depresión, ansiedad y asertividad. Revista Mexicana de Psicología, 18 (1), 150.
Franklin, C. \& Streeter, C. L. (1995). Assessment of middle class youth at-risk to dropout: School, psychological and family correlates. Children and Youth Services Review, 7 (3), 433-448.

Frydenberg, E. (1997). Adolescent coping. Theoretical and Research Perspectives. New York. Routledge.

Harter, S. (1983). Developmental perspectives on the self-system. En E. M. Hetherington (Ed.), Handbook of Child Psychology (4th ed.). Socialization, personality, and social development (Vol. 4, pp. 443 460). Nueva York: Wiley.

Hair, J. F, Anderson, R. E., Jr., Tathman, R. L. \& Black, W. C. (1999). Análisis Multivariante. Madrid: Pearson Educación.

Hoge, D. R., Smit, E. K. \& Crist, J. T. (1995). Reciprocal effects of self-concept and academic achievement. Journal of Youth and Adolescence, 24 (3), 295-314.

Jiménez, J. \& Yáñez, S. (2006, septiembre). Autoestima en adolescentes: análisis de consistencia interna y estructura factorial. Memorias del XIV Congreso Mexicano de Psicología, Puerto Vallarta, México.

Joreskog, K. \& Sorbom, D. (2001). Lisrel 8 User's Reference Guide. Lincolnwood, Illinois: Scientific Software International, Inc.

Linacre, J. M. (2007). Winsteps (Version 3.62.3) [Computer Software]. Chicago: Winsteps.com

Litten, K. (1999). A prevention program design for inner-city, high school adolescents to combat learned helplessness and depression. Dissertation Abstracts International, 60 (3-B), 1306.

Nunnally, J. C. \& Bernstein, I. H. (1994). Psychometric Theory. New York: Mc Graw-Hill.

Shavelson, R. J., Hubner, J. J. \& Stanton, G. C. (1976). Self-concept: Validation of construct interpretations. Review of Educational Research, 46, 407-441.

Tristán, A. \& Vidal, R. (2006). Manual de fórmulas de correlación. México: Editorial Trafford. 
